\title{
Élaborer un savoir sur la sexualité : le Dictionnaire des sciences médicales (1812-1822)
}

The Construction of Sexual Knowledge: The Dictionnaire des sciences médicales (1812-1822)

\section{Clyde Plumauzille}

\section{OpenEdition}

Journals

\section{Édition électronique}

URL : http://journals.openedition.org/clio/9611

DOI : 10.4000/clio.9611

ISSN : $1777-5299$

Éditeur

Belin

Édition imprimée

Date de publication : 1 mai 2010

Pagination : 111-132

ISSN : 1252-7017

Référence électronique

Clyde Plumauzille, «Élaborer un savoir sur la sexualité : le Dictionnaire des sciences médicales

(1812-1822) », Clio. Femmes, Genre, Histoire [En ligne], 31 | 2010, mis en ligne le 23 août 2013, consulté le 19 avril 2019. URL : http://journals.openedition.org/clio/9611; DOI : 10.4000/clio.9611 


\section{Élaborer un savoir sur la sexualité : le Dictionnaire des sciences médicales (1812-1822) ${ }^{1}$}

Clyde Plumauzille

«Le plaisir est une sensation que l'on éprouve, mais que l'on ne définit pas. Comme une vapeur légère, il s'envole dès qu'on veut l'analyser. L'Homme du monde en jouit sans le connaittre; le Philosophe le connoît, \& ne peut l'approfondir $»^{2}$.

Dans Les Réflexions philosophiques sur le Plaisir de Grimaud de la Reynière, le plaisir, sensation évanescente, a ses raisons que la raison ignore. État d'âme de l'instant, il participe de l'idée de bonheur que le XVIII ${ }^{\mathrm{e}}$ siècle a développée avec intensité. C'est son flottement conceptuel et la liberté sensuelle qui en découle que les médecins modernes tentent d'appréhender, de fixer et de déterminer dans l'ordre de la Nature à une époque charnière où les bouleversements révolutionnaires entraînent dans leur sillage une forte politisation du sexuel. Les savoirs médicaux qui entendent alors être un instrument essentiel du diagnostic social participent pleinement à cette dynamique $^{3}$. Ils constituent des lieux d'investigation incontournables pour comprendre les multiples façons dont s'élabore la sexualité, comprise comme les pratiques sexuelles et les normes qui prétendent régir ces pratiques. Aussi, alors que le mot même n'a pas encore trouvé son entrée propre dans la nomenclature scientifique ${ }^{4}$, la

1 Je tiens à remercier chaleureusement Jean-Luc Chappey, Nathalie Richard et Sylvie Steinberg pour leurs lectures et conseils avisés.

2 Grimaud de la Reynière $1783: 17$.

3 Blanckeart 2000/2:118.

4 Le mot fera son apparition en 1837 dans la traduction française d'un livre du biologiste Karl-Friedrich Burdach. Cf. Corbin 2008 : 17. On parle alors d'« acte 
médecine du XVIII ${ }^{\mathrm{e}}$ siècle entreprend néanmoins d'en décrire les différentes modalités. Deux lignes de force se dégagent de ces études. La première est la fixation des identités de sexe et leur naturalisation comme l'a démontré l'étude pionnière de l'historienne Yvonne Knibiehler ${ }^{5}$ à propos de la définition médicale de la «nature féminine». La seconde, récemment étudiée par Alain Corbin dans L'harmonie des plaisirs, est la codification par la médecine des pratiques sexuelles afin de "rationaliser le mystère de la jouissance $»^{6}$. Ces leitmotivs du discours scientifique renvoient plus largement à l'attention essentielle que porte alors la société aux pratiques corporelles et sexuelles. Selon Michel Foucault, c'est au cours du XVIII $^{e}$ siècle que le sexe tend à constituer un objet d'analyse scientifique générant une véritable «explosion discursive » notamment dans les milieux savants 7 . Dans le cadre de cette «volonté de savoir ", il met en lumière l'émergence d'un dispositif de sexualité qui établit des liens de causalité entre désir et identité personnelle, faisant de la sexualité un élément clé de la subjectivité moderne ${ }^{8}$. Cette notion de dispositif est particulièrement intéressante pour notre étude du traitement encyclopédique de la sexualité dans le Dictionnaire des sciences médicales qui paraît au début du XIXe siècle. Elle permet de souligner l'importance du système formel et éditorial qui contribue alors à l'élaboration et à la mise en ordre d'un savoir sur la sexualité.

\section{Le renouveau du naturalisme}

\section{et l'émergence d'une physiologie de la sexualité}

L'émergence d'une physiologie de la sexualité est à mettre en relation avec le «moment naturaliste» du second XVIII ${ }^{e}$ siècle identifié par Claude Blanckeart qui redéfinit l'homme comme un «produit naturel» et tente de comprendre les déterminismes généraux de l'évolution du genre humain. Buffon, figure tutélaire du

vénérien » (voir l'article «Jouissances (anticipées) », Dictionnaire des sciences médicales 1818, vol. $26: 417$. Désormais DSM).

5 Knibiehler 1976.

6 Corbin $2008: 17$.

7 Foucault 2004 [1976].

8 Ibid. : 163-164. 
naturalisme, rédige une histoire naturelle de l'homme en 1749 où il opère une analyse descriptive de l'espèce humaine et dégage les lois générales de son évolution'. Il pose également les jalons d'une physiologie de la sexualité qui insiste sur les cadres naturels et matériels de la sexualité reproductive, faisant du plaisir une expérience subjective et organique, et de l'attraction sexuelle entre homme et femme un résultat de leur dimorphisme sexué ${ }^{10}$. L'histoire de l'espèce humaine prend un nouvel essor à la fin du siècle avec les productions des médecins vitalistes et physiologistes de l'Institut et de son organe de presse, La Décade. L'idéologue et médecin Pierre-Jean-Georges Cabanis présente ainsi entre 1795 et 1806 une douzaine de mémoires dans Les Rapports du physique et du moral chez l'homme et la femme où il entend "démontrer invinciblement la grande influence des sexes sur la formation des affections morales et des idées $»^{11}$. À propos des «habitudes morales», il insiste sur «l'influence des organes de la génération et des fonctions qui s'y rapportent » dans le tempérament humain car, écrit-il, «ces fonctions et ces organes exercent un empire étendu non seulement sur la production des penchants heureux de l'amour, de la bienveillance, de la tendre et douce sociabilité, mais encore sur l'énergie et l'activité de tous les autres organes particulièrement de l'organe pensant ou du centre nerveux principal $»^{12}$. La prégnance des organes sexuels est fonction de leur "sensibilité singulière », ce qui selon Cabanis est " conforme aux lois de l'économie animale ${ }^{13}$.

Dans cette perspective, avec les travaux des médecins et des naturalistes Roussel, Cabanis, Moreau de la Sarthe ou Virey ${ }^{14}$, on assiste à la mise en lumière, dans le champ scientifique, d'une anthropologie des sexes et de la sexualité fondée sur des enquêtes empiriques ${ }^{15}$. Ces enquêtes constituent également le fer de lance du projet de régénération porté par la «République des sciences » qui

\footnotetext{
9 Buffon $2006: 33$.

10 Hoffmann $1995: 99-100$.

11 Cabanis 1844 [1802] : 256.

12 Ibid. : 357

13 Ibid. : 226.
}

14 Roussel 1775 ; Cabanis 1802 ; Moreau de la Sarthe 1803 ; Virey 1801, 1823.

15 Blanckeart 2000/2: 127. 
entend changer l'homme lui-même pour mener à son terme le progrès social et politique et le rendre irréversible. En d'autres termes, il s'agit d'appliquer le concept politique de révolution au domaine des mœurs entendu ici comme l'ensemble des comportements humains. Le journaliste et écrivain Pierre-Louis Ginguené dans son article publié dans la Décade sur la première séance publique de l'Institut, insiste ainsi sur l'utilité de l'étude menée par Cabanis qui «donnera enfin à la morale des fondements solides ${ }^{16} »$, permettant par cette connaissance des lois de la nature un progrès moral et physique de l'humanité.

Outre cet arrière-plan intellectuel, le dynamisme de la presse savante participe également du renouvellement du savoir sur la sexualité ${ }^{17}$. Sur le plan éditorial, le premier XIX ${ }^{\mathrm{e}}$ siècle constitue un moment de renouveau pour l'encyclopédisme qui joue à la fois sur la juxtaposition des différents savoirs dans les périodiques et la spécialisation fine des disciplines dans le cadre de dictionnaires. C'est alors qu'est édité l'imposant Dictionnaire des sciences médicales chez l'éditeur Panckoucke ${ }^{18}$. Si la médecine constitue le cœur de ce dictionnaire, elle n'est pas isolée du reste des sciences naturelles. Ainsi les théories du mouvement vitaliste qui émanent de l'ensemble des naturalistes - et non des seuls médecins -, y occupent une large place. Pour eux, la spécificité du vivant réside dans l'organisation, sorte de «tout fonctionnel $»^{19}$ comprenant le corps et ses sensations. Dans le domaine de la physiologie, a contrario du dualisme cartésien, ils insistent sur les rapports entre le moral et le physique chez l'individu et sur une conception de la santé - c'est-à-dire de l'état physiologique normal-, fondée sur les principes d'équilibre ${ }^{20}$ et d'économie d'énergie ${ }^{21}$. Aussi peut-on lire dans le Dictionnaire que «c'est au médecin que les hommes doivent la conservation du plus précieux de tous leurs biens, la santé ». Dans ce cadre de réflexion, la médecine

\footnotetext{
16 Boulad-Ayoub $2003: 6$.

17 Rabier 2004.

18 Knibiehler $1976: 826$.

19 Wenger $2005: 3$.

20 Jin-Han $2008: 29$.

21 Stengers \& Van Neck 1998 : 109.
} 
s'intéresse aux usages physiques et imaginaires du sexe considéré comme un objet physiologique et moral dont il convient également de définir l'équilibre. De nombreux articles sont ainsi consacrés à des sujets aussi divers que la "Copulation», le "Libertinage», le «Plaisir» ou encore le «Génital», la «Jouissance», la «Masturbation», la « Prostitution ».

La place accordée à ces sujets est d'autant plus significative que le Dictionnaire fut une véritable plateforme de diffusion pour les autorités scientifiques de l'époque. L'éditeur Charles-Louis-Fleury Panckoucke fit en effet appel à des sommités, comme Alibert, Pinel, Esquirol, Laennec, Desgenettes ou Larrey afin d'offrir une synthèse du savoir médical à la naissance de la clinique et de l'anatomie-pathologie. Son réseau de collaborateurs est constitué de praticiens en exercice, tous liés et intégrés aux institutions hospitalières et universitaires parisiennes ce qui signifie que leurs articles sont le résultat d'observations pratiques. Il s'agit d'une nouvelle génération de médecins qui furent les élèves de l'École de Médecine de Paris, fondée en 1795 sous l'impulsion des Idéologues comme Vicq d'Azyr ou Cabanis.

Du point de vue de sa diffusion, cette édition monumentale de 60 volumes in- $8^{\circ}$ (35 000 pages) parus entre 1812 à 1822 fut un véritable succès de librairie. Premier dictionnaire médical encyclopédique du XIX ${ }^{e}$ siècle, avant ceux de Jaccoud (1864-1886) ${ }^{22}$ et Dechambre (1864-1889) ${ }^{23}$ dont il constitue une référence théorique fondatrice, sa diffusion s'étend à l'ensemble de l'élite intellectuelle et savante de l'époque ${ }^{24}$. Cet outil de "vulgarisation de haut niveau » se veut aussi un manuel d'observations pratiques pour les médecins, les officiers de santé et les étudiants ${ }^{25}$. Ainsi la section «Pratique» de l'article «Médecin » rédigé par Jean-Baptiste Monfalcon, médecin hospitalier à l'Hôtel-Dieu puis à la Charité de Lyon, détaille tout le protocole d'observation et d'interrogation qui intervient dans la rencontre entre le médecin et son patient et indique comment celui-ci établit ses

\footnotetext{
22 Jaccoud 1864-1886.

23 Dechambre 1864-1889.

24 Knibiehler $1976: 826$.

25 Edelman $2003: 21$.
} 
prescriptions à l'issue de ce «colloque singulier» ${ }^{26}$. L'«art d'observer» est primordial dans cette démarche ${ }^{27}$ qui rejoint la préoccupation des naturalistes de fonder les théories scientifiques sur un empirisme analytique ${ }^{28}$. Monfalcon précise en outre que, " quoique les principes de la médecine soient constants, il est souvent difficile d'en faire l'application aux cas particuliers ${ }^{29}$. C'est pourquoi les auteurs du Dictionnaire tentent le plus souvent d'opérer un aller-retour entre la théorie médicale et leurs observations pratiques. Virey à propos de l'endurance sexuelle féminine affirme qu'il tient « de l'aveu d'une femme (moins intéressée qu'un homme à surfaire en ce genre) qu'elle compta onze actes complets du même homme en une nuit » et qu'une « femme de débauche (...) s'abandonna en une nuit à vingt-etun soldats $»^{30}$. Fournier dans son article "Masturbation», se réfère également à l'un de ses collègues : «l'un de nous a connu une petite fille qui, dès l'âge de quatre ans, se livrait comme par instinct à la masturbation $»^{31}$.

\section{L'encyclopédisme médical et la mise en discours du sexuel}

L'ensemble des connaissances contenues dans le dictionnaire sont aussi le résultat de croyances et de représentations sociales sur la sexualité. Il s'agit de la vision propre à une médecine officielle, reconnue et soutenue par l'État. Les contributeurs, en activité dans les facultés et les hôpitaux, le sont dans des institutions placées sous la tutelle du gouvernement. Napoléon avait instauré un contrôle fort de l'État dans le domaine de l'éducation et de la pratique médicale par le biais de la refondation de facultés de médecine intégrées à la nouvelle Université impériale et la fondation d'un Conseil général d'administration des hôpitaux de Paris ${ }^{32}$. Rien d'étonnant à ce qu'on trouve dans le dictionnaire des références élogieuses au Code civil

\footnotetext{
26 Monfalcon, art. « Médecin », DSM 1819, vol. 32 : 289-293.

27 Ibid. : 289.

28 Corsi $2005: 233$.

29 Monfalcon, art. « Médecin », DSM 1819, vol. 32 : 290.

30 Virey, art. «Femme », DSM 1815, vol. 14 : 539.

31 Fournier \& Begin, art. « Masturbation », DSM 1819, vol. 31 : 102.

32 Williams 2002 : 113-114.
} 
napoléonien et à «ses sages dispositions $»^{33}$. Par ailleurs, comme l'entreprise napoléonienne de codification qui, selon la juriste Marcela Iacub, a fait de la sexualité "non seulement un ensemble de comportements, mais aussi une matrice de production des espaces institutionnels $»^{34}$, les définitions médicales et la prétention encyclopédique du Dictionnaire à l'égard de la sexualité répondent à une volonté de fixer le lien social et sexuel. Savoir et Pouvoir s'articulent donc dans cette mise en discours des plaisirs. Les auteurs ne renoncent pas néanmoins à se positionner en dehors du champ médical: leur anthropologie des plaisirs se veut aussi une métaphysique des mœurs, une pensée philosophique de la libido et de son inscription morale dans la société. Ainsi, l'étude de «l'acte reproducteur» ne constitue pas uniquement une entreprise scientifique mais participe de l'ambition philosophique et morale de cette science de l'homme que Cabanis avait annoncée: le médecin doit être «le surveillant de la morale, comme de la santé publique $»^{35}$. Prétention que l'on retrouve également chez les naturalistes comme Virey qui se réclame, dans son article «Libertinage», d'« une médecine morale et philosophique ${ }^{36}$, une médecine qui «trace encore la route de la morale au milieu même des plaisirs $»^{37}$. Virey s'inscrit ici dans la tradition des médecins philosophes qui ont entrepris au cours des dernières décennies du XVIII ${ }^{\text {e }}$ siècle d'établir des «systèmes physiques et moraux » des individus, véritable fabrique médicale des corps sexués où le sexe biologique détermine les rôles et les qualités morales des hommes et des femmes en société. Les médecins entendent édicter des «lois de l'économie animale» au niveau de l'individu et tracer celles de l'hygiène publique collective qu'elles garantissent ${ }^{38}$. La conviction des hygiénistes qu'il existe un rapport inéluctable entre le physique et le moral, un lien entre la forme de l'individu et sa fonction sociale, entre son âme et son corps

\footnotetext{
33 Geoffroy, art. «Copulation », DSM 1813, vol. 6 : 264.

34 Iacub $2008: 24$.

35 Cabanis $1819: 146$.

36 Virey, art. «Libertinage », DSM 1818, vol. 28 : 113.

37 Virey 1823-1825: V.

38 Virey, art. «Jouissances (anticipées) », DSM 1818, vol. $26: 417$.
} 
fait de la sexualité un enjeu de régulation et de protection du corps social. Théories vitalistes et hygiénistes se recoupent ainsi, générant une véritable philosophie médicale de la sexualité valable à la fois pour les individus et pour le corps social.

La modernité du propos réside ici moins dans le fond que dans la forme. Le Dictionnaire par son caractère encyclopédique occupe une position centrale dans l'espace savant du XIXe siècle. L'unité synthétique de cette médecine morale est garantie par le label encyclopédique de l'ouvrage et la notoriété de son éditeur. Si les cadres théoriques héritent en grande partie du siècle précédent, l'objet pratique et sa fonction changent d'horizon dès lors que ce savoir se trouve reformulé. Les travaux actuels de Jean-Luc Chappey sur les usages de l'encyclopédisme insistent sur l'importance du dispositif formel de la littérature encyclopédique du premier XIXe siècle ${ }^{39}$. La forme encyclopédique peut ainsi offrir un outil efficace pour codifier et unifier de nouveaux savoirs. Le Dictionnaire permet par son découpage en articles et par leur classement suivant les différentes spécialités disciplinaires - « Anatomie », «Anthropologie », « Hygiène publique », "Morale »...-, un traitement varié des formes et des normes de la sexualité qui se constitue comme un objet transversal. Mais plus encore, c'est par la pratique de la citation et le système de renvoi que le Dictionnaire élabore une mise en relation et une mise en système de diverses analyses et théories. Ce système de renvoi constitue un outil important de la forme encyclopédique, établissant des connexions logiques entre des notices anatomiques, physiologiques et morales. Il offre ainsi des dossiers thématiques et des lignes directrices pour la lecture, ce qui contribue à unifier le savoir médical sur la sexualité.

39 Chappey Jean-Luc, 2009, Les usages de l'encyclopédisme dans la presse savante entre le XVIII et le XIXe siècle, journée d'étude organisée par Institut d'histoire de la Révolution française / Ea 127/ Ums 622 / Université Paris I PanthéonSorbonne, le CIM Communication Information Médias / Ea 1484 / Université Paris III Sorbonne-Nouvelle et le Centre Alexandre Koyré / Centre de recherche en histoire des sciences et des techniques UMR 8560 du CNRS, Université Paris I Panthéon-Sorbonne, 27 novembre 2009. Non publiée. 


\section{Dépense libidinale et énergie vitale}

La question qui traverse l'ensemble des articles est celle de la gestion du plaisir. Selon Virey, l'espèce humaine dans ses caractères physiques semble être destinée à la sexualité et à ses «plus ardents plaisirs » et, ajoute-t-il, « la nature accroît ce penchant et augmente les moyens de jouissance, à mesure qu'on se rapproche de l'espèce humaine $»^{40}$. L'homme, "plus favorisé de tous pour les voluptés $»^{41}$, se distingue des autres espèces animales par la supériorité de son désir et de ses pratiques. À en croire l'article «Impuissance $»^{42}$, le plaisir et l'absence de plaisir sont en outre déterminés par des causes morales, car le psychique, et plus particulièrement l'imagination, joue un rôle essentiel dans l'appréhension de la sexualité. Dans son article «Génital», Rullier affirme ainsi qu'«il n'est point d'organes qui ressentent plus puissamment que les parties génitales l'influence des affections morales et des idées $»^{43}$. "L'effet de l'imagination, par exemple, se montre dans sa toute puissance » et l'excitation sexuelle dérive en grande partie de celle-ci. Le plaisir sexuel se définit ainsi par cette « sympathie » des organes génitaux et du cerveau.

La libido vient donc complexifier la description de l'acte sexuel qui relève d'une fonction vitale nécessaire au corps humain et à son épanouissement, mais est également une source de plaisirs. La sexualité constitue la source des plaisirs par excellence, plaisirs qui « rassemblent dans un point et dans un instant toutes les forces de la vie qu'ils multiplient et qu'ils éternisent $»^{44}$. Cependant pour Bilon ${ }^{45}$, si les plaisirs sont nécessaires à l'épanouissement de l'organisme, les jouissances physiques et mentales doivent être exercées en accord avec "l'intégrité organique et vitale», ce qui pose problème car la «satisfaction des sens » ne semble obéir à aucune règle ${ }^{46}$, si ce n'est à une pulsion instinctive qui échappe à tout contrôle social ou médical. Les médecins admettent ainsi «l'existence d'un tempérament génital,

\footnotetext{
40 Virey, art. «Libertinage », DSM 1818, vol. 28 : 118.

41 Ibid. : 117.

42 Marc, art. «Impuissance », DSM 1818, vol. 24 : 180-181.

43 Rullier, art. « Génital », DSM 1817, vol. 18 : 126-127.

44 Ibid. : 133.

45 Bilon, art. « Plaisir », DSM 1820, vol. 43 : 124-132.

46 Geoffroy, art. «Copulation », DSM 1813, vol. $6: 288$.
} 
qui communément subordonné, acquiert souvent aussi une indépendance licencieuse, et parfois une prédominance insurmontable $»^{47}$. Les sensations sexuelles constituent ainsi un des éléments clefs du tempérament des individus et les organes génitaux possèdent un potentiel libidinal et une autonomie problématiques du point de vue de l'organisme.

La critique de la dépense libidinale sous ses aspects économiques, culturels et physiques parcourt l'ensemble des articles et rejoint la crainte de l'excès et de "l'addiction" qui pourraient s'attacher à certaines formes de sexualité. Thomas Laqueur dans Le sexe en solitaire a souligné cette caractéristique du discours médical à l'égard de l'onanisme dans le courant du XVIII ${ }^{\mathrm{e}}$ siècle $^{48}$. Au fil des notices du dictionnaire, l'excès des plaisirs devient une caractéristique de la sexualité en général. L'imagination joue à ce niveau un rôle délicat car elle exerce un effet prégnant sur les organes génitaux et l'accouplement selon l'article qui lui est consacré. Celui-ci ${ }^{49}$ au détour d'une présentation du rôle de l'imagination dans les lois de l'attraction sexuelle renvoie d'ailleurs à l'article "Génération ». Mais c'est bien évidemment le problème de la masturbation qui se joue également dans la relation entre plaisir et imagination: question de santé publique tout au long du XVIII ${ }^{\mathrm{e}}$ siècle, elle est aussi un symptôme critique de l'incontinence sensuelle. Dans l'article «Masturbation» qui cite abondamment les travaux de Tissot, on peut lire que «la tension extrême de l'imagination» et la facilité d'une pratique «de tout instant $\aleph^{50}$, résultent en une fabrique artificielle et surtout illimitée de plaisirs. Cette forme de satisfaction sexuelle entraîne un certain repli égoïste sur la jouissance personnelle qui ne devrait manifestement pas avoir sa place dans l'espace public impérial, collectif et dépassionné dont «le gouvernement de soi» devrait garantir l'équilibre. De plus, selon Virey, l'énergie investie dans la jouissance, prive la société de la vigueur de ses individus dont «la force nerveuse ou sensitive» est littéralement «épuisée par les

\footnotetext{
47 Bilon, art. « Plaisir », DSM 1820, vol. 43 : 132.

48 Laqueur $2005: 272$.

49 Virey, art. «Imagination », DSM 1818, vol. 24 : 26.

50 Fournier \& Begin, art. « Masturbation », DSM 1819, vol. 31 : 118.
} 
voluptés vénériennes ». Le parallélisme entre l'énergie sexuelle du corps individuel et l'énergie vitale du corps social revient sans cesse dans la rhétorique hygiéniste comme si cette rhétorique pouvait permettre l'encadrement du désir sexuel à l'échelle de la société.

\section{Le libertinage, un risque physiologique, moral et social}

Dans ses articles "Jouissances anticipées » et «Libertinage », Virey fait le lien entre l'économie des plaisirs et celle de sa santé : «Il n’y a point de courage et de santé sans bonnes mœurs ${ }^{51}$. Le plus «important précepte » pour perfectionner le capital énergétique viril est la continence car le sperme est une « source de vigueur vitale» et, «conservé dans l'économie animale»-entendue ici comme organisme -, il rend l'homme «plus viril »52. Si les plaisirs sexuels restent nécessaires «à la satisfaction du bien être » ${ }^{53}$, il n'en demeure pas moins que «pour jouir, les tissus vivants ne doivent être ni trop ni point assez stimulés» car l'excès sensuel menace l'intégrité organique des individus, ce qui est «en contradiction avec notre nature $\aleph^{54}$. L'énergie vitale et les capacités physiques opposent donc des limites à une libido excessive. Selon Geoffroy, une consommation excessive de «boissons fortes », de partenaires sexuels, un « désir trop vif de procréer », et des «passions violentes», sont en outre autant de causes de la stérilité55. La jouissance est affaire de vitalité et il faut être «capable de supporter la dépense de forces que le coït exige " $^{56}$. Virey, dote ainsi son article «Libertinage » d'un renvoi aux articles «Énergie», «Esprit», et "Génie », où il explique «que la force nerveuse ou sensitive [est] principalement épuisée par les voluptés vénériennes, laissant le cerveau incapable de penser, comme les muscles deviennent incapables de forts mouvements $»^{57}$. La sexualité s'inscrit donc dans une économie libidinale dont les savants fixent les

\footnotetext{
51 Virey, art. « Libertinage », DSM 1818, vol. 28 : 113.

52 Virey, art. «Énergie », DSM 1815, vol. $12: 212$.

53 Bilon, art. « Plaisir », DSM 1820, vol. 43 : 124.

54 Ibid. : 128.

55 Geoffroy, art. « Copulation », DSM 1813, vol. 6 : 246.

56 Ibid. : 276.

57 Virey, art. « Libertinage », DSM 1818, vol. 28 : 144.
} 
principes afin que l'homme puisse accomplir sa destinée physique et morale, et conserver force, intelligence et longévité.

L'économie libidinale est d'autant plus nécessaire que se dresse la «débauche» qui met en péril et "énerve la force virile», et fait «éluder le but de la copulation» aux «femmes libertines $»^{58}$. La sexualité sans autre finalité que le plaisir génère aux yeux des médecins une déviance de la nature: l'homme se retrouve affaibli voire impuissant et la femme en vient à nier son rôle maternel. Soulignons ici qu'il est bien plus question du risque encouru par la gent masculine que par la gent féminine car comme nous le verrons, les femmes sont censées être plus résistantes aux excès du coït. Chez les hommes, d'une part, une consommation précoce et excessive entraîne un affaiblissement organique, d'autre part leurs descendants mâles risquent d'être eux-mêmes affectés par cet affaiblissement, tendant à constituer "des générations ignobles et flasques », des « individus grêles rabougris, sans énergie, sans cervelle » et surtout des « êtres efféminés ${ }^{59}$. L'« énervement » que génère l'excès provoque la "fureur» chez les femmes et la «faiblesse» chez les hommes, inversant la partition passive/active du genre des individus féminins/masculins.

Par ailleurs, la quête effrénée des plaisirs peut également constituer un problème d'ordre public. Tous les articles insistent sur le fait qu'elle renforce la «prostitution» et la propagation du «mal vénérien ». Si la prostitution est perçue comme "un mal nécessaire » dans les grandes villes, Virey et Geoffroy réclament une administration policière "éclairée et sévère » des maisons closes pour contenir la prostitution et en appellent à «des mesures administratives propres à diminuer cette légèreté ». Ils citent le Pornographe (1769) de Restif de la Bretonne qui faisait de l'encadrement de la prostitution par le gouvernement une affaire de bien public. Restif préconisait même l'enfermement des prostituées dans des sortes de "maisons-prisons" afin qu'elles entretiennent le moins de contact possible avec la cité. Les médecins, quant à eux, craignent de voir les hommes exposés «aux caresses empoisonnées

58 Geoffroy, art. « Copulation », DSM 1813, vol. 6 : 288.

59 Virey, art. « Libertinage », DSM 1818, vol. 28 : 113. 
de la première venue $»^{60}$. Les prostituées - femmes uniquement forment une classe infectieuse et dangereuse. Des mesures administratives sont réclamées à l'encontre des «dangers vénériens » qui, selon Geoffroy, "s'opposent au mariage » et à la copulation ${ }^{61}$. " À cet égard, nos lois positives ne sont pas aussi sévères qu'en ma qualité de médecin je me vois forcé de l'être ${ }^{62}$, note-t-il. Au passage, les médecins encensent la police napoléonienne qui « a fait à cet égard un grand pas vers le bien; car depuis quelques années on [ne] voit plus de femmes à moitié nues se pencher hors des croisées et attirer en plein jour les passants par des sifflements impudiques $»^{63}$. L'article «Prostitution» revient également sur la nécessité d'isoler géographiquement les prostituées, insiste sur l'importance de leur enregistrement auprès de la police à Paris et de leur surveillance médicale ${ }^{64}$.

Ce discours s'inscrit pleinement dans la politique de réglementation de la prostitution apparue en France sous le premier Empire et codifiée sous la Restauration, qui place les prostituées sous la tutelle administrative de l'État. C'est moins la prostitution que sa visibilité qu'il convient d'endiguer. Si les médecins reconnaissent qu'il n’existe pas de véritables "préservatifs » aux maladies vénériennes, leur dramatisation permet de proposer une administration du sexe dans la société, établissant ainsi les jalons théoriques d'un véritable «biopouvoir» qui, pour Michel Foucault, marque «l'entrée des phénomènes propres à la vie de l'espèce humaine dans l'ordre du savoir et du pouvoir ${ }^{65}$ et «dans le champ des techniques politiques $»^{66}$. Les notices du Dictionnaire témoignent des cadres de la régulation médicale du corps social visant à une mise en ordre des plaisirs à partir d'une redéfinition de la sexualité à l'aune de la « santé ».

\footnotetext{
60 Geoffroy, art. «Copulation », DSM 1813, vol. 6 : 297.

61 Ibid. : 272.

62 Ibid. : 266.

63 Geoffroy, art. «Copulation », DSM 1813, vol. 6 : 297.

64 Podéré, art. «Prostitution », DSM 1820, vol. 45 : 490.

65 Foucault 1994 : 181

66 Ibid. : 186.
} 


\section{Hétérosexualité et « art de copuler »}

Le dictionnaire offre aussi une définition positive des bonnes mœurs sexuelles. Les médecins dressent des limites pour que les jouissances «ne soient point nuisibles à l'intégrité de l'organisation animale, mais encore qu'elles ne soient pas contraires au maintien de l'ordre social $»^{67}$. Si la sexualité participe de la nature humaine, c'est que «l'acte vénérien » est avant tout un «acte reproducteur» pour Bilon. Dans le sillage des discours populationnistes du XVIII siècle, les médecins insistent sur le caractère nécessaire de la sexualité pour l'accroissement de l'espèce humaine. Du fait de la reproduction, le rapport sexuel est «ce qui donne à l'existence sa valeur ${ }^{68}$. Les médecins définissent ainsi une forme politique et sociale de la sexualité, conçue comme hétérosexuelle, conjugale et reproductrice ; l'association sexuelle entre les sexes ne se trouve justifiée que dans le cadre du mariage qui constitue selon Geoffroy ${ }^{69}$ "la copulation autorisée par les lois». Dans l'article «Femme», Virey au regard de son étude de «l'état naturel» des différentes «races» humaines et animales, affirme que «tout parait annoncer que la femme et l'homme doivent, en nombre égal, concourir à former la famille $»^{70}$. Le traitement médical de la sexualité se veut le prétexte à l'élaboration d'un ordre biologique, moral et social qui assigne des rôles sexuels aux individus en société : l'«homme animal», doit ainsi user des plaisirs en accord avec les lois de "l'homme intelligent», de l'«homme individuel» et de l' «homme social » ${ }^{71}$. C'est dans cette perspective, que la définition de la sexualité joue sur la fabrique « naturelle » des sexes comme le souligne Elsa Dorlin qui voit le genre être «déterminé par la sexualité, comprise comme système politique, en l'occurrence l'hétérosexualité reproductive, qui définit le féminin et le masculin par la polarisation sexuelle socialement organisée des corps $»^{72}$.

\footnotetext{
67 Bilon, art. « Plaisir », DSM 1820, vol. 43 : 129.

68 Ibid. : 132.

69 Geoffroy, art. «Copulation », DSM 1813, vol. $6: 244$

70 Virey, art. «Femme », DSM 1815, vol. $14: 531$.

71 Ibid. : 130.

72 Dorlin $2008: 55$.
} 
Dans le prolongement de la théorie des humeurs où les différences sociales reproduisent les différences de tempéraments ${ }^{73}$, les «tempéraments sexuels» établissent un clivage selon le sexe des individus. Virey remarque ainsi, à propos des «forces naturelles de l'homme et de la femme dans l'acte vénérien», que «la femme, en général résiste plus longuement à des entreprises masculines » ${ }^{74}$. Cette endurance n'est pas le fait d'une supériorité physiologique car « si l'on demande pourquoi la femme se montre plus insatiable que l'homme dans les plaisirs de l'amour, nous croyons que c'est parce qu'elle dépense moins ». Il renvoie alors à l'article «Génération» pour expliquer les dispositions féminines à recevoir l'acte sexuel. Cette posture passive de réceptacle à l'action énergique masculine reflète la différenciation sexuée et sexuelle des individus, hommes et femmes. Nous avons vu dans l'article «Énergie» du même auteur que l'homme se définissait essentiellement par son potentiel énergétique. Il est intéressant de remarquer que l'article «Femme » en revanche porte essentiellement sur la vie sexuelle et sensible des femmes.

Le plaisir, ses manifestations et sa gestion, participent ainsi pleinement de la différenciation homme/femme. Il est aussi un principe essentiel à l'«harmonie des sexes» et les médecins de demander régulièrement une meilleure administration des relations sexuelles. Des mesures sont ainsi suggérées pour prévenir les unions mal assorties ${ }^{75}$. Les articles rendent compte des critères qui doivent entrer en ligne de compte, notamment l'âge, le physique et la moralité des individus, car la sexualité est affaire de "nubilité », de "vigueur » - c'est-à-dire de maturité physique et morale - et de contrôle. La maturité est fixée arbitrairement à «quelques années» après la puberté soit aux alentours de 18 ans. Critère plus social que véritablement biologique, cet âge de la nubilité (âge légalement requis afin de pouvoir contracter mariage) est également défini par le Code civil à 15 ans pour les filles et 18 ans pour les garçons. Pour les médecins, il convient d'éviter les passions violentes de la jeunesse pour que «l'attachement [prévale] sur la sensualité» et que «les

73 Steinberg $2001: 280$.

74 Virey, art. « Femme », DSM 1815, vol. 14 : 539.

75 Geoffroy, art. « Copulation », DSM 1813, vol. 6 : 267. 
époux [dirigent] leurs efforts communs vers le perfectionnement physique et moral de la nouvelle génération $»^{76}$. Cette arithmétique physique vise une fois de plus à contenir les excès libidinaux des individus. Par ailleurs, et c'est ce qui justifie le choix de cette limite d'âge, les « unions précoces » constituent selon Virey et Geoffroy une des causes de l'affaiblissement de la race humaine puisque les individus qui n'ont pas atteint leur pleine puissance physique ont une « semence » incomplète.

Dans son article sur la copulation, Geoffroy dresse, en outre, une typologie des copulations stériles et dangereuses qui nuisent à son «but essentiel», la procréation ${ }^{77}$. Le critère positif qui semble prédominer est la vitalité et la vigueur des partenaires. Les malades, les handicapés et les fous ne constituent pas des partenaires viables et leur état empêche le plein accomplissement de la sexualité. L'« harmonie des sexes » est ainsi perçue comme le prélude nécessaire à l'harmonie de la sexualité. Par ailleurs, on décèle une certaine anxiété à l'égard de l'hérédité dangereuse et morbide que portent ces individus à risque. Ce problème de l'hérédité, déjà abordé à l'occasion de la question de la dépense énergétique, est alors un leitmotiv du discours médical populationniste. La parole médicale élabore ainsi un véritable «art de copuler» conforme à la nature en général et à celle, supposée, de chaque sexe. Elle catégorise et hiérarchise les individus selon des critères de différenciation comme le sexe, les caractères physiques et l'âge. La classification physique qu'elle établit peut être perçue à cet égard comme un projet de normalisation des individus en société.

\section{Naturaliser les plaisir, stabiliser la société}

Le discours des médecins qui écrivent dans le dictionnaire participe de la tentative plus générale qu'opère alors la société postrévolutionnaire de stabiliser la société. On ne peut ignorer l'opération de lissage des comportements individuels entrepris par les différents codes napoléoniens qui visent à discipliner une société dont on souhaite gommer le caractère révolutionnaire. Ce retour à l'ordre

76 Ibid. : 255.

77 Ibid. : 244. 
intervient dans un contexte d'«après» Révolution. La crise révolutionnaire qui résonne encore profondément dans l'esprit des auteurs - qui en ont été le plus souvent des contemporains-, a généré une grande incertitude quant à l'expression et au sens des hiérarchies sociales. Selon Denise Davidson, «dans le sillage de cette expérience déstabilisatrice, un nouvel ensemble de distinctions sociales commençait à émerger» et, au sein de cette confusion, les distinctions de genre prirent un rôle significatif « car le genre semblait plus "naturel" que les autres catégories de différenciation ${ }^{78}$. La "Nature est à l'ordre du jour"

L'usage de la sexualité est ainsi perçu comme le reflet de la vertu des gouvernements car «le code de la morale privée et celui de la morale publique reposent sur les mêmes bases; que l'une et l'autre sont voulues par la nature qui les a dictées $»^{80}$. Virey dénonce ainsi les systèmes politiques qui reposent sur la débauche: système de cour d'un Ancien Régime libertin, système du harem des despotes orientaux. Il reprend le leitmotiv de la licence de l'Ancien Régime et fait de la Révolution la victoire du Tiers vertueux et vigoureux sur des aristocrates libertins et dégénérés ${ }^{81}$. La Révolution aurait ainsi été le produit d'une sorte de « sélection naturelle», de «lutte des races $»^{82}$ entre l'ancienne aristocratie défaillante et la nouvelle bourgeoisie énergique. Il s'inscrit ici dans tout un discours médical qui, à la fin du XVIII ${ }^{e}$ siècle, insiste sur le fait que la dégénérescence de l'espèce humaine n'est pas le fait de la nature mais de la société83. L'attention portée à la question de la sexualité et à sa "naturalité" participe d'une volonté déjà présente chez les hygiénistes des Lumières de perfectionner l'espèce ${ }^{84}$. C'est en référence à cette interprétation

78 Davidson $2007: 2$.

79 Nous reprenons ici le titre de l'ouvrage de Colette Capitan, La nature à l'ordre du jour, 1789-1793, Paris, Kimé, 1993.

80 Bilon, art. «Plaisir », DSM 1820, vol. 43 : 130.

81 Virey, art. « Libertinage », DSM 1818, vol. 28 : 141-142.

82 Hannah Arendt, dans son chapitre consacré à la pensée raciale avant le racisme, parle du combat qui oppose une « race » d'aristocrates contre une «nation » de citoyens, Arendt [1954] $2002: 80-86$.

83 Steinberg $2001: 286$.

84 Ibid. : 289. 
naturaliste et "naturalisante" de la société que les médecins tentent de dégager un état de nature sexuel originel et vertueux où l'homme doit être maitre et gestionnaire de ses besoins.

Ce contexte particulier - la quête de repères stables et l'essor de la jeune anthropologie qui fait de l'espèce humaine un objet d'observation et d'expérience - explique la tendance des auteurs à façonner une norme médicale d'organisation et de gestion des besoins sexuels. Les articles comportent de nombreuses références à Rousseau et plus encore aux travaux des naturalistes sur les autres espèces animales. Virey cite ainsi ses propres travaux sur la génération parus dans son Nouveau dictionnaire de sciences naturelles afin de saisir les causes de la «lubricité ou de la lascivité » chez les animaux « comparés à l'homme $»^{85}$. Il opère de la même façon dans son article «Génération » et établit que «les délices de l'amour» sont fonction de la complexité du système nerveux ${ }^{86}$. Il avait d'ailleurs proposé dès 1803 une méthode de classement des êtres vivants en faisant du système nerveux le critère absolu de la distribution animale ${ }^{87}$. La complexité du système nerveux atteint son apogée chez l'espèce humaine qui "possède au plus haut degré l'attribut également précieux et pernicieux d'une extrême sensibilité, au physique comme au moral ${ }^{88}$. En dépit de sa supériorité, l'espèce humaine demeure assujettie aux mêmes règles de la Nature et ce particulièrement dans le domaine problématique de la sexualité. Dans son article "Jouissance », Virey insiste bien sur la communauté de destin entre humain et animal, assurant qu'«il est manifeste qu'on ennoblit l'espèce humaine et les races d'animaux en retardant leur génération » et que, de la même façon, « rien ne rabougrit, n'abâtardit, n'avilit plus les races que la multiplicité prématurée des reproductions, qui énerve les individus pour accroître leurs plaisirs». Aussi «les racailles abâtardies » des grandes cités sont issues des mêmes «voluptés prématurées » que les races naines d'animaux ${ }^{89}$. La polygamie

\footnotetext{
85 Virey, art. « Libertinage », DSM 1818, vol. 28 : 114.

86 Virey, art. «Génération », DSM 1817, vol. 18 : 14-15.

87 Benichou \& Blanckeart 1992 : 9.

88 Virey, art. «Libertinage », DSM 1818, vol. 28 : 119.

89 Virey, art. «Jouissances (anticipées) », DSM 1818, vol $26: 418$.
} 
humaine et animale a pour semblable conséquence la génération d'un plus grand nombre de progénitures femelles, signe de l'abâtardissement de la race. L'unique rempart à cette crise est la conservation et la multiplication de "jeunes mâles vigoureux », seuls garants de la régénération de leur race ${ }^{90}$. Ce parallèle entend inscrire les êtres humains comme le reste des animaux dans un "ordre de la Nature » qui détermine des pratiques « naturelles » de la sexualité.

La naturalité de la sexualité participe toujours d'une volonté de fixer les identités à partir de la corrélation entre les dispositions de nature et le désir sexuel. La vigueur des individus masculins constitue la clef de voûte de l'édifice. Ils doivent gérer leur "capital physique" afin de conserver le système physique et moral de la société tout entière. Si nous avons pu voir que le corps et le diktat de sa santé constituaient un premier rempart à la dépense libidinale, la Nature fixe aussi des contraintes et des limites à la sexualité. Par une sorte de raisonnement cyclique, la sexualité, produit de la Nature, est également contrainte et encadrée par elle. Ces contraintes ont été soulignées par Yvonne Knibiehler dans son étude de la «nature féminine » à partir de l'article «Femme» du Dictionnaire des sciences médicales qui vient « rajeunir une longue tradition de sujétion féminine, en lui apportant des justifications neuves $»^{91}$. Il nous faut également souligner l'ensemble des contraintes qui régissent l'injonction à la virilité et à la vigueur pour les hommes. Ceux-ci sont menacés d'être « moins masculins » et « moins ardents $»^{92}$ s'ils dérogent au modèle de la virilité guerrière qui s'est imposée tout au long du premier XIX ${ }^{\mathrm{e}}$ siècle. Le coût physique du plaisir constitue une remise en cause du genre masculin puisque les hommes sont susceptibles en permanence de s'affaiblir et donc de s'efféminer ${ }^{93}$. On retrouve ces «images inquiètes de la sexualité masculine» dont parlait Alain Corbin dans

\footnotetext{
$90 \quad$ Ibid. : 119-120.

91 Knibiehler $1976: 845$.

92 Virey, art. «Jouissances (anticipées) », DSM 1818, vol. $26: 419$.

93 On reprendra ici les analyses de Sylvie Steinberg du terme «effémination » qui « indique que l'artifice règne dans la société, suivant l'analyse rousseauiste selon laquelle l'influence des femmes a civilisé les hommes à l'excès ». Cette effémination c'est aussi la « dénaturation» du sexe masculin qui « met en péril leur capacité d'engendrement», Steinberg $2001: 288$.
} 
son essai sur Le "sexe en denil» et l'histoire des femmes ${ }^{94}$. Toute une rhétorique à la tonalité moraliste et injonctive entend ainsi restructurer la sexualité selon un principe d'autorité, qui n'est plus religieux mais médical, rejoignant le leitmotiv idéologique du gouvernement napoléonien qui a réorganisé la société révolutionnaire selon ce même principe : autorité de l'État qui s'exprime par une administration centralisée, hiérarchisée et efficace, autorité du père de famille dont le Code civil consacre la domination sur l'ensemble des membres de son foyer, autorité du chef d'entreprise sur ses employés associée au contrôle policier, par le biais du livret ouvrier.

Les faisceaux de discours qui se tissent dans le dictionnaire opèrent ainsi une véritable "naturalisation" des pratiques et des imaginaires sexuels. La naturalité biologique et sociale fait de la sexualité une donnée en soi, vraie et authentique, qui ordonne les rapports entre les sexes et les façons de "faire du sexe". Le plaisir n'est plus seulement une simple sensation évanescente telle que la décrivait Grimaud de la Reynière puisqu'il se retrouve strictement encadré par la Nature et la Société. «Les affections de l'âme ne sont la source des vrais plaisirs que lorsqu'elles sont conformes à notre nature; qu'elles concourent à maintenir la régularité dans la vie et l'ordre dans la société ${ }^{95}$, écrit Bilon. En filigrane de cette rhétorique moraliste et naturaliste, on décèle un souci d'ordre qui traverse alors la France postrévolutionnaire et renouvelle les politiques de gestion et de surveillance des conduites. Les médecins contribuent à conceptualiser et naturaliser les cadres modernes de l'hétérosexualité triomphante de la période contemporaine. Une réglementation hygiénique et raisonnable résume la sexualité à une pratique efficace, orientée vers la procréation. Ni les folies érotiques de l'imaginaire, ni son parfum d'infini et son usage intime, n'ont le droit de cité dans une taxinomie où la sexualité est un plat qui se mange froid. Le discours n'est certes pas novateur, dès lors qu'il se trouve déjà dans l'ensemble des écrits médicaux du XVIII ${ }^{e}$ siècle. La véritable modernité de cette pensée réside plus dans les cadres de sa diffusion.

\footnotetext{
94 Corbin $1991: 93$.

95 Bilon, art. « Plaisir », DSM 1820, vol. 43 : 130.
} 
Il ne s'agit plus d'écrits isolés mais d'articles qui prennent place dans le premier dictionnaire encyclopédique médical. C’est le positionnement de ce Dictionnaire dans le champ éditorial comme ouvrage de référence rassemblant les plus grands théoriciens et praticiens issus de la révolution médicale de la fin du XVIII ${ }^{\mathrm{e}}$ siècle qui fait toute la nouveauté de cet ensemble théorique. L'anthropologie des sexes par sa présence importante dans le Dictionnaire, est ainsi partie prenante de la modernité médicale alors en élaboration. On ne peut s'empêcher de voir dans l'unification des discours au sein de ce dictionnaire encyclopédique et dans le croisement des rubriques "Anatomie », «Hygiène », " Physiologie », « Morale », les prémices de l'organisation d'un savoir sur la sexualité. Le Dictionnaire crée une sorte d'unité fictive des savoirs sur la sexualité dans la circulation qui se joue entre les articles. Par ailleurs, la large diffusion de cet ouvrage qui touche autant le public médical que l'élite savante de l'époque offre un caractère plus central à la sexualité dans le champ scientifique et une ventilation plus large de ces théories auprès du public. Ces théories ne constituent certes pas encore un savoir expérimental comme le sera la sexologie du second XIXe siècle mais la modernité de leur écriture et de leur diffusion est déjà en acte.

\section{Sources}

Buffon, 2006, De l'Homme. Buffon, présentation par Michèle Duchet, postface de Claude Blanckaert, Paris, L'Harmattan.

CABAnis Pierre-Jean-Georges, 1819 (3e éd.), Du degré de certitude de la médecine, Paris, Caille et Ravier.

—, 1844 [1802], Rapports du physique et du moral de l'homme, Paris, Fortin, Masson \& cie.

Dechambre Amédée (éd.), 1864-1889, Dictionnaire encyclopédique des sciences médicales, Paris, G. Masson, P. Asselin.

Grimaud DE LA REYNière, 1783, Réflexions sur le plaisir par un célibataire, Neufchatel et Paris, Veuve Duchesne, Le Jay, Desenne, Petit.

Dictionnaire des sciences médicales, 1812-1822, Paris, Panckoucke, 60 vol.

JACCOUD (éd.), 1864-1886, Nouveau dictionnaire de médecine et de chirurgie pratiques, illustré de figures intercalées dans le texte, Paris, J.-B. Baillière. 
Moreau de la SARTHE Jacques-Louis, 1803, Histoire naturelle de la Femme, suivie d'un traité d'bygiène appliqué à son régime physique et moral aux différentes époques de sa vie, Paris, L. Duprat, Letellier et Cie, 2 vol.

ROUSSEL Pierre, 1775, Système physique et moral de la femme ou Tablean philosophique de la constitution, de l'état organique, du tempérament, des mours, \&o des fonctions propres au sexe, Paris, Vincent.

VIREY Julien-Joseph, 1801, Histoire naturelle du genre humain, Paris, Dufart, 3 vol.

—, 1823-1825, De la femme sous ses rapports physiologique, moral et littéraire, Paris, s.e.

\section{Ouvrages cités}

ARENDT Hannah, 2002 [1954], L'Impérialisme. Les origines du totalitarisme, Paris, Seuil.

Benichou Claude \& Claude BlanCKAERT, 1992 [1988], Julien-Joseph Virey. Naturaliste et Anthropologue, Paris, Vrin.

Blanckeart Claude, 2000/2, «1800 - Le moment "naturaliste” des sciences de l'homme », Revue d'histoire des sciences humaines, 3, p. 117-160.

Boulad-Ayoub Josiane (éd.), 2003, La Décade philosophique comme système. Sciences philosophiques, morales et politiques. 1794-1798, t. V, Rennes, Presses Universitaires de Rennes.

CAPITAN Colette, 1993, La nature à l'ordre du jour, 1789-1793, Paris, Kimé.

CORBIN Alain, 1991, Le temps, le désir et l'horreur, Paris, Aubier.

—, 2008, L'harmonie des plaisirs. Les manières de jouir du siècle des Lumières à l'avènement de la sexologie, Paris, Perrin.

CORSI Pietro, 2005, «After the Revolution: scientific language and French politics, 1795-1802 », in M. Pelling \& S. Mandelbrote (eds), The Practice of Reform in Health, Medicine, and Science, 1500-2000, Aldershot, Ashgate, p. 1-23.

Davidson Denise, 2007, France after Révolution. Urban Life, Gender, and the New social order, Cambridge (Mass.), Harvard University Press.

DorLin Elsa, 2008, Sexe, genre et sexualités, Paris, PUF.

Edelman Nicole, 2003, Les métamorphoses de l'bystérique. Du début du XIXe siècle à la grande guerre, Paris, La Découverte.

Foucault Michel, 2004 [1976], Histoire de la sexualité, t. I : La volonté de savoir, Paris, Gallimard.

Hoffmann Paul, 1995, La femme dans la pensée des Lumières, Genève, Slatkine.

IACuB Marcela, 2008, Par le trou de la serrure. Une histoire de la pudeur publique (XIXXXIe siècle), Paris, Fayard. 
Élaborer un savoir sur la sexualité : le Dictionnaire des sciences médicales 133

JiN-Han Hee, 2008, « Georges Canhuilhem et le vitalisme français : remarques sur les "Aspects du vitalisme" ", in Anne Fagot-Largeault, Claude Debru \& Michel Morange (éd.), Philosophie et médecine: en hommage à Georges Canguilhem, Paris, Vrin, p. 13-34.

KNIBIEHLER Yvonne, 1976, "Les médecins et la "nature féminine" au temps du Code civil », Annales. Économies, Sociétés, Civilisations, 31, 4, p. 824-845.

LAQUEUR Thomas, 2005, Le sexe en solitaire, contribution à l'bistoire culturelle de la sexualité, Paris, Gallimard.

RABIER Christelle, 2004, "Vulgarisation et diffusion de la médecine pendant la Révolution : l'exemple de la chirurgie », Annales historiques de la Révolution française, 338, p. 75-94.

SAAD Mariana, 2000, «La médecine constitutive de la nouvelle science de l'homme : Cabanis », Annales historiques de la Révolution française, 320, p. 55-64.

STEINBERG Sylvie, 2001, La confusion des sexes. Le travestissement de la Renaissance à la Révolution, Paris, Fayard.

Stengers Jean \& Anne VAN NECK, 1998, Histoire d'une grande peur, la masturbation, Paris, Pocket.

WENGER Alexandre, 2005, «Lire l'onanisme. Le discours médical sur la masturbation et la lecture féminine au XVIII" siècle », Clio. Histoire, femmes et sociétés, 22, p. $227-$ 243.

Williams Elizabeth A., 2002, The Physical and the Moral: Anthropology, Physiology, and Philosophical Medicine in France, 1750-1850, Cambridge, Cambridge University Press. 